\title{
e-Phaïstos
}

e-Phaïstos

Revue d'histoire des techniques / Journal of the history

of technology

IX-2 | 2021

Quel objet pour quel musée?

\section{Le chemin de fer Noroeste do Brasil et les paysages industriels de l'Ouest de l'État de São Paulo}

Patrimoine de la mobilité au Brésil

Mobility Heritage in Brazil: The Noroeste do Brasil Railway and the Industrial Landscapes of Western São Paulo State

\section{Taís Schiavon}

\section{OpenEdition}

Journals

Édition électronique

URL : https://journals.openedition.org/ephaistos/9053

DOI : 10.4000/ephaistos.9053

ISSN : 2552-0741

Éditeur

IHMC - Institut d'histoire moderne et contemporaine (UMR 8066)

\section{Référence électronique}

Taís Schiavon, «Le chemin de fer Noroeste do Brasil et les paysages industriels de l'Ouest de l'État de São Paulo », e-Phaïstos [En ligne], IX-2 | 2021, mis en ligne le 26 octobre 2021, consulté le 28 octobre 2021. URL : http://journals.openedition.org/ephaistos/9053 ; DOI : https://doi.org/10.4000/ephaistos. 9053

Ce document a été généré automatiquement le 28 octobre 2021

Tous droits réservés 


\section{Le chemin de fer Noroeste do Brasil et les paysages industriels de l'Ouest de l'État de São Paulo}

Patrimoine de la mobilité au Brésil

Mobility Heritage in Brazil: The Noroeste do Brasil Railway and the Industrial

Landscapes of Western São Paulo State

Taís Schiavon

1 Fruit de la recherche effectuée dans le cadre du master Erasmus Mundus TPTI (Schiavon 2015), cet article s'inscrit également dans la continuité d'études effectuées au

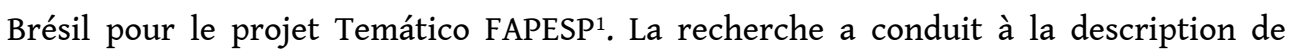
l'occupation d'environ $65 \%$ du territoire de l'État de São Paulo (Brésil) à partir de l'avancement de "la frange pionnière » décrite par le géographe français Pierre Monbeig (1908-1987). Ce dernier a étudié le réseau de villes fondées avec l'avènement du chemin de fer dans les « régions inconnues et habitées par les Indiens » (Salgueiro 2006), représentant la région ouest de l'État de São Paulo.

2 La mise en relation de nos recherches avec l'Association pour l'histoire des chemins de fer (AHICF) à partir de 2015, a encouragé une circulation des connaissances, des informations historiques et des données territoriales, ainsi qu'un élargissement vers de nouvelles frontières d'études. Confortant cette approche, notre recherche a reçu en 2015 et 2017 une reconnaissance internationale après avoir été classée parmi les finalistes pour le prix «CILAC jeunes chercheurs» (Paris, France, 2015) et pour le «III Premio Aula G+I_PAI » (Madrid, Espagne, 2017). En 2018, la publication de notre mémoire est intervenue, sous la forme d'un livre traduit en portugais (Schiavon 2018).

3 Notre objectif est d'insérer cette étude dans un projet qui vise à la création d'une "Collection Patrimoniale Virtuelle ${ }^{2}$ », structurée à partir d'un "réseau », capable d'identifier, numériser et systématiser le territoire urbain traversée par les voies du chemin de fer (de la partie ouest de l'État de São Paulo, y compris le chemin de fer Noroeste do Brasil, objet de cette recherche), dans une perspective passée et présente. 


\section{Objectif}

4 Le chemin de fer peut être identifier comme l'élément moteur du processus d'urbanisation de la région Ouest de l'État de São Paulo, au Brésil. Les influences de sa construction s'exercent dans un contexte national et international, étant donné que le tracé du chemin de fer Noroeste do Brasil faisait partie d'un tronçon en quête d'une communication entre les océans Atlantique et Pacifique; le Brésil étant le principal acteur dans le contexte sud-américain. Il s'agit d'étudier cette réalisation et le paysage urbain qu'elle a fait naître comme un moyen de valorisation du Patrimoine Industriel et du Patrimoine de la Mobilité au Brésil, à partir de l'analyse des différents centres urbains dispersés le long de l'axe ferroviaire de la partie Ouest de l'État de São Paulo ${ }^{3}$.

Fig.1. Principales Villes de l'État de São Paulo

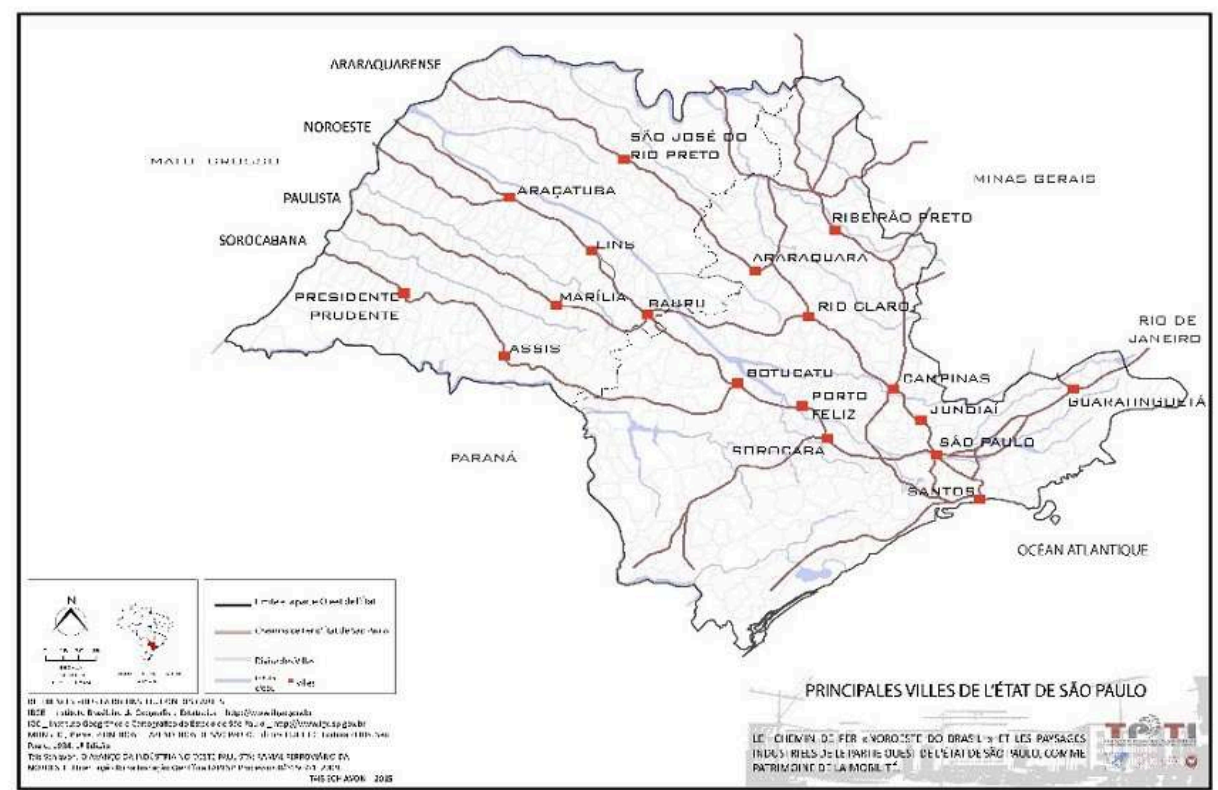

La carte montre le positionnement des principales villes de l'État de São Paulo au Brésil et leur chevauchement avec le réseau ferroviaire de l'État, consolidé tout au long du XXe siècle.

Source : Schiavon 2005. Réalisé par l'auteur à partir des bases consultées

Le chemin de fer Noroeste do Brasil fut créé en 1905 au milieu de «l'arrière-pays » de la région Ouest de l'État São Paulo, où un complexe de villes s'est développé à partir de réseaux tout au long de l'avancée des lignes. En plus d'ouvrir «l'arrière-pays ", le chemin de fer entraîne le processus de construction et de qualification des villes. On observe, principalement dans les villes nommées «bouches de l'arrière-pays 4 ", l'apparition d'activités directement liées à la voie ferrée, susceptibles d'être associées à la nouvelle dynamique née de la présence du chemin de fer. Les relations nationales et internationales peuvent être considérées comme un aspect caractéristique de cette nouvelle organisation urbaine. À ce titre, de nombreux ingénieurs européens se rendent au Brésil pour effectuer les travaux nécessaires à la création et au fonctionnement du chemin de fer. 


\section{Problème}

En moins de dix ans, la voie ferrée Noroeste do Brasil parvient à mettre en communication les États de São Paulo et Mato Grosso (actuellement Mato Grosso do Sul). De cette façon, le chemin de fer est devenu la première voie de connexion et, par conséquent, le premier moyen de dissémination technique, technologique et industrielle dans toute cette région.

7 Après son installation, une série d'autres activités industrielles ont été implantées grâce à l'avancée ferroviaire. Ainsi, les villes « créées » par le chemin de fer ont profité d'un réseau d'infrastructures urbaines qui ont modifié considérablement le paysage précédent. Grâce à ce transfert transocéanique des connaissances, la logique d'organisation urbaine des villes ouvertes par l'avancée du «front pionnier ${ }^{5}$ » était comparable à celle des grandes villes brésiliennes, mais aussi européennes.

Fig.2. Bauru, siège de la compagnie de chemin de fer Noroeste do Brasil : chemins de fer et autoroutes

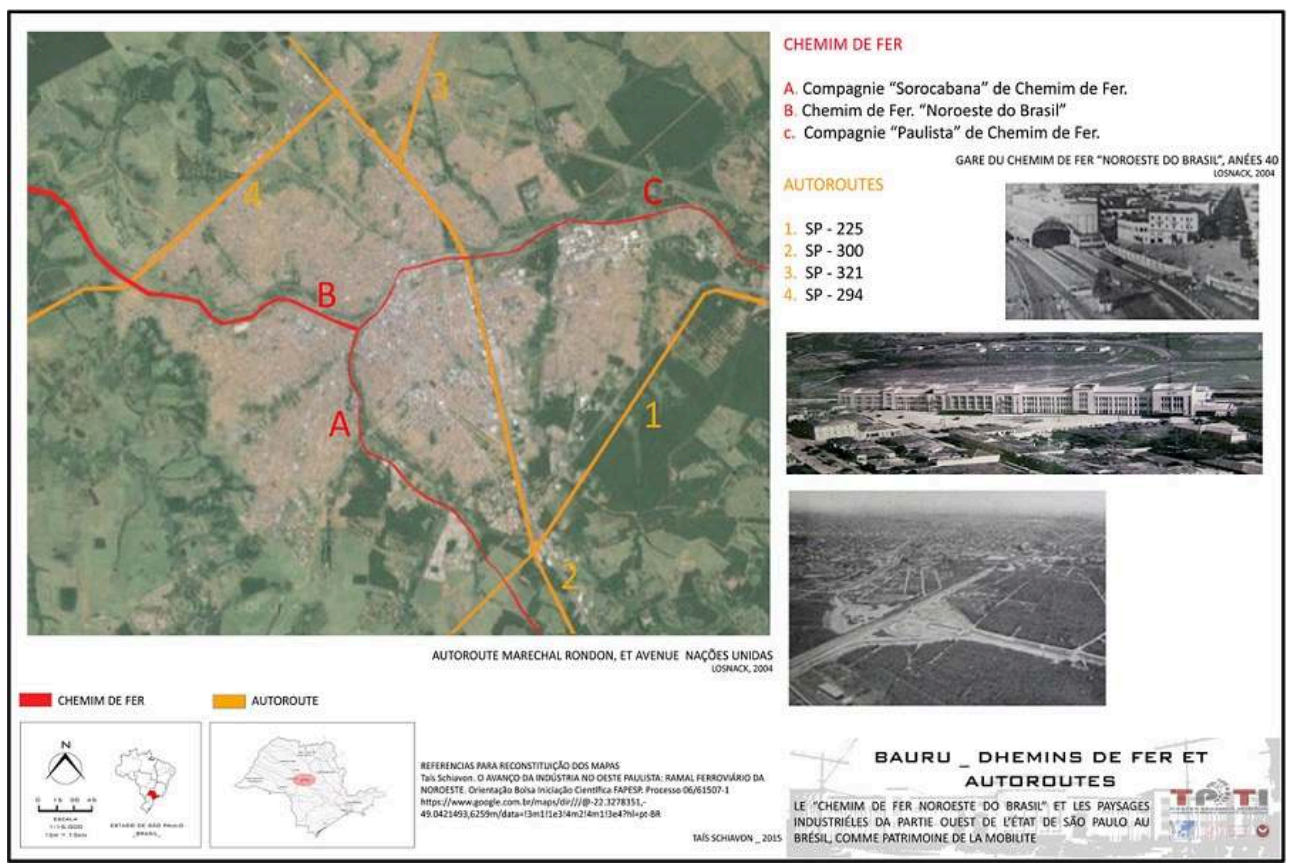

La zone urbaine actuelle de la ville de Bauru, siège de la compagnie de chemin de fer Noroeste do Brasil, dans la partie ouest de l'État de São Paulo, Brésil. En superposition de l'image satellite,

l'itinéraire des compagnies ferroviaires qui ont coupé la ville est indiqué en rouge: A- Compagnie Sorocabana de chemin de fer ; B- Chemin de fer Noroeste do Brasil ; C- Compagnie Paulista de chemin de fer. Les principales autoroutes qui traversent la commune sont indiquées en orange : 1 -SP-225 ; 2 SP-300 ; 3-SP321; 4-SP-204. Les photographies soulignent l'importance de l'emprise des points modaux construits dans la ville.

Source : Schiavon 2005. Réalisé par l'auteur à partir des bases consultées

8 Le caractère transcontinental de la ligne émerge vers 1908, à une époque où l'activité des autres compagnies de chemin de fer brésiliennes commence à se contracter. En revanche, le chemin de fer Noroeste do Brasil fait l'objet d'un grand enthousiasme. Pour la deuxième fois, l'Océan Atlantique devient l'endroit idéal pour le mouvement et la navigation des connaissances et des innovations, ainsi que pour la réévaluation du Brésil, considéré au $\mathrm{XX}^{\mathrm{e}}$ siècle comme un endroit propice à l'étude et à la pratique 
professionnelle. Ce contexte nous permet d'identifier l'histoire et les personnages impliqués dans ce double mouvement entre une ex-colonie et les puissances européennes. D'une part, les étrangers viennent au Brésil pour découvrir le pays et, d'autre part, les professionnels brésiliens cherchent en Europe des connaissances techniques à transférer (Karvar 1994, Bresciani 2009).

La compréhension des politiques d'installation des moyens de transport dans le contexte économique et spatial brésilien, surtout quand on se réfère à la région Ouest de l'État de São Paulo, montre que le complexe urbain développé à partir du modèle ferroviaire puis la transition vers le modèle routier de transports, à partir des années 1930, provoquent sur le « réseau » de villes une rupture physique. À partir des années 1970 et 1980, la plupart des régions où les infrastructures ferroviaires sont développées sont abandonnées en raison de la fermeture des gares et de la disparition des compagnies ferroviaires (Prado 2011).

Fig.3. Bauru : le pátio ferroviaire et ses environs

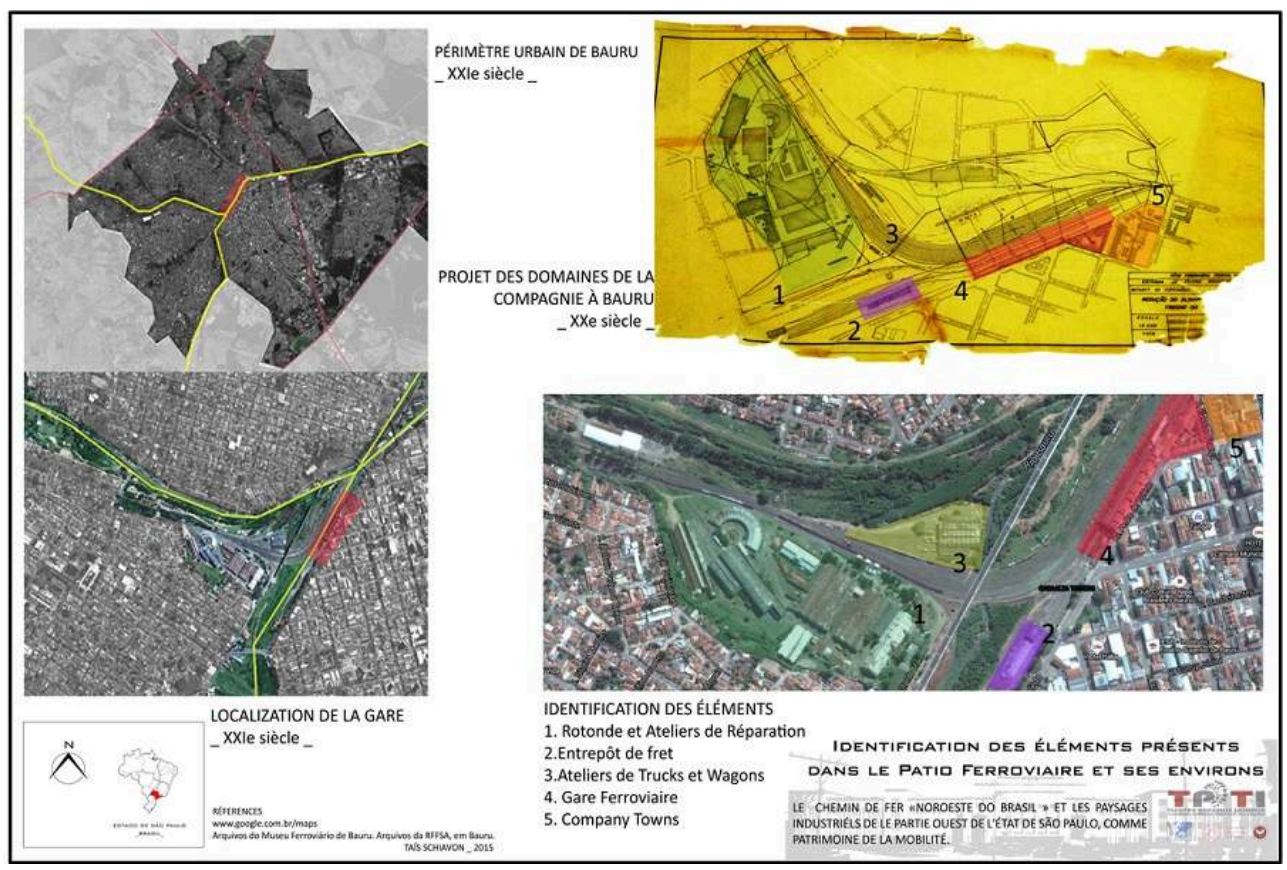

La figure montre le chevauchement des axes ferroviaire (jaune) et autoroutier (rouge), mettant en évidence l'emplacement du Chemin de fer Noroeste do Brasil. Sont mises en évidence : la rotonde et les ateliers de réparation (1) ; l'entrepôt de fret (2) ; les ateliers pour les wagons et boggies (3) ; la gare (4) ; et la cité ouvrière (5)

Source : Schiavon 2005. Réalisé par l'auteur à partir des bases consultées

10 L'intérêt de cette recherche vise à comprendre l'agencement des villes, afin d'analyser les effets liés à la composition du maillage territorial dans la transition du modèle ferroviaire au modèle routier et d'interroger également les possibilités et potentialités de réhabilitation.

«(...) Aujourd'hui c'est le chemin de fer le fondateur des villes et l'élément qui favorise, avec l'industrie, les grandes agglomérations urbaines (...) aujourd'hui villes de premier ordre (...) ${ }^{6}{ }^{\prime}$ (Azevedo 1950) (traduit par l'auteur). 


\section{Méthodologie} une série de transformations. Notre analyse méthodologique est fondée sur le principe des enquêtes historiques et géographiques à partir des villes traversées par les axes des compagnies du chemin de fer. Les caractéristiques techniques ne sont pas uniquement liées à l'axe ou à une ville spécifique, mais plutôt portées par l'articulation de villes organisées comme une sorte de réseau linéaire déterminé par le chemin de fer principal, mais aussi avec d'autres connexions situées perpendiculairement à l'axe majeur?

Fig.4. État de São Paulo : les villes concernées par la recherche

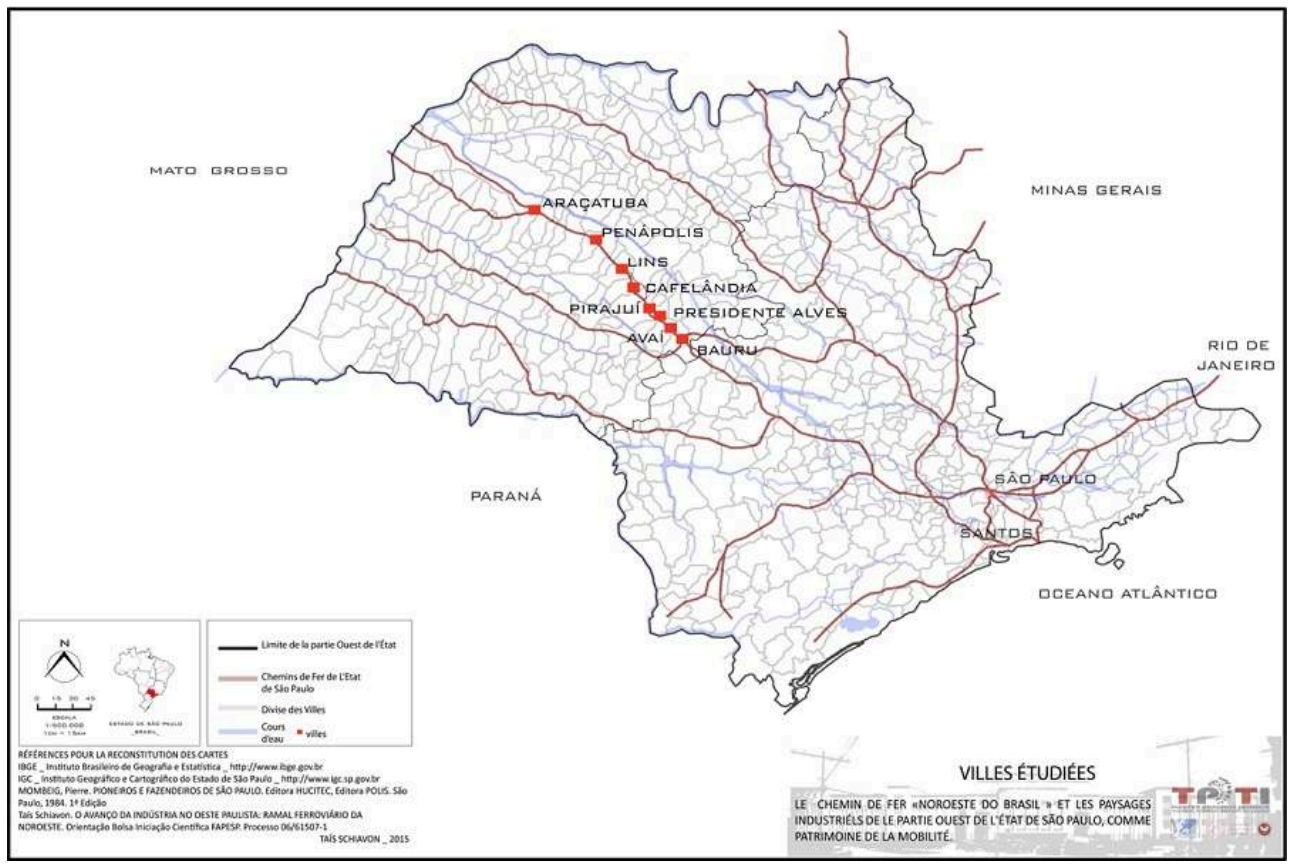

La carte met en évidence les villes étudiées par la recherche : Buru, Avaí, Presidente Alves, Pirajuí, Cafelândia, Lins, Penápolis, Araçatuba. Ces villes chevauchent le tracé du chemin de fer nord-ouest du Brésil, dans la partie ouest de l'État de São Paulo.

Source : Schiavon 2005. Réalisé par l'auteur à partir des bases consultées

Les concepts fondés autour de la mobilité permettent d'aborder la transformation du paysage naturel, dans lequel le chemin de fer devient l'élément responsable de la formation d'un patrimoine industriel complexe.

La recherche a développé des moyens de catalogage et de cartographie afin d'identifier et décrire les types fonctionnels trouvés dans chaque ville. Différentes échelles d'analyse ont été développées. Le croisement de données régionales et locales autorise l'étude du développement de la région Ouest de l'État de São Paulo ${ }^{8}$, mais aussi de 
l'évolution ferroviaire du Brésil', en particulier à partir du chemin de fer Noroeste do Brasil.

Considérant le binôme "Chemin de Fer \& Industrie » et la répercussion de ses étapes sur le contexte urbain, cette étude valorise la notion de «Patrimoine Industriel » telle que le définit le TICCIH dans sa Charte de Nizhny Tagil :

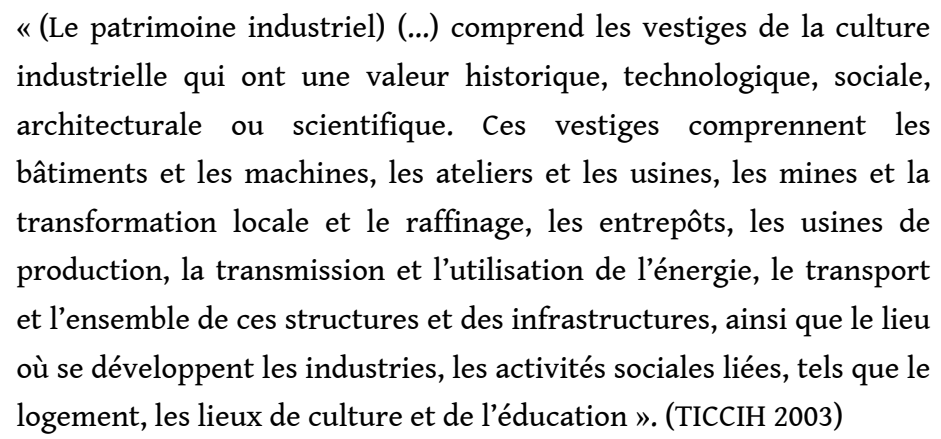

$\mathrm{Au}$ Brésil, ces concepts ont été appliqués à l'inspection menée par l'IPHAN ${ }^{10}$, dans la mesure où le patrimoine généré par les compagnies de chemins de fer est similaire à celui imposé par les industries. En ce qui concerne la notion des chemins de fer comme patrimoine, l'IPHAN a commencé son approche au Brésil à partir de la promulgation de la loi 11483 en $2007^{11}$.

\section{La dispersion ferroviaire au Brésil}

17 Le cas brésilien démontre la forte présence des investissements internationaux, en particulier anglais, français et belges, et l'insertion du pays dans un système international de circulation et de dispersion de la connaissance et de la technique, associés à la formation de "professionnels modernes ». Le passage du XIX ${ }^{\mathrm{e}}$ au XXe siècle voit se développer, autour du chemin de fer, une nouvelle forme d'occupation et de valorisation du sol. Dans son étude classique sur l'Ouest de l'État, Monbeig parle de «franges pionnières », pour caractériser les zones qui ont d'abord été ouvertes par les chemins de fer (Monbeig 1984). Bien avant les vagues successives de pionniers du XX $\mathrm{X}^{\mathrm{e}}$ siècle, une grande partie du pays était encore inconnue en effet (Salgueiro 2006), comme l'indique clairement la carte dressée dans les années 1910/1912, qui mentionne « un territoire inexploré habité par les Indiens ${ }^{12}$ » (Retto 2011, traduit par l'auteur).

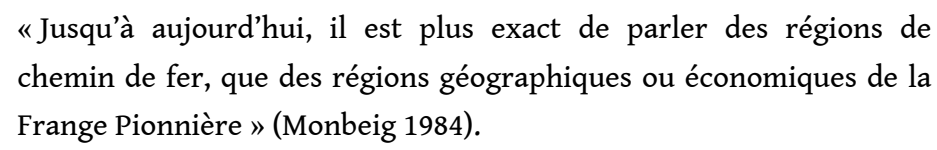

18 Jusque-là, l'exploitation des ressources naturelles représentait la plus importante activité économique. De ce processus ont résulté plusieurs axes pour répondre aux difficultés de communication entre les zones productrices et les marchés consommateurs. Les stratégies et les plans d'occupation du territoire ont donc recherché à promouvoir la communication entre ces différentes régions (Pinto 1903).

19 La "marche vers l'Ouest", dans la seconde moitié du XIX ${ }^{e}$ siècle, coïncide avec la colonisation du territoire et l'ouverture de nouvelles zones de culture, provoquée par la réduction de la production du café dans l'État du Rio de Janeiro. Les chemins de fer 
prennent alors en charge la circulation des produits de l'intérieur vers les ports, définissant des trajets connus sous le terme de "couloirs d'exportation». Si l'on considère que $65 \%$ du territoire de l'État de São Paulo a été urbanisé à partir de l'avancée de la "frange pionnière ", en relation avec la production du café, nous comprenons l'importance de la performance des compagnies ferroviaires au Brésil, surtout entre le XIX et le XX ${ }^{e}$ siècles.

Fig.5. État de São Paulo : l'évolution de la "frange pionnière"

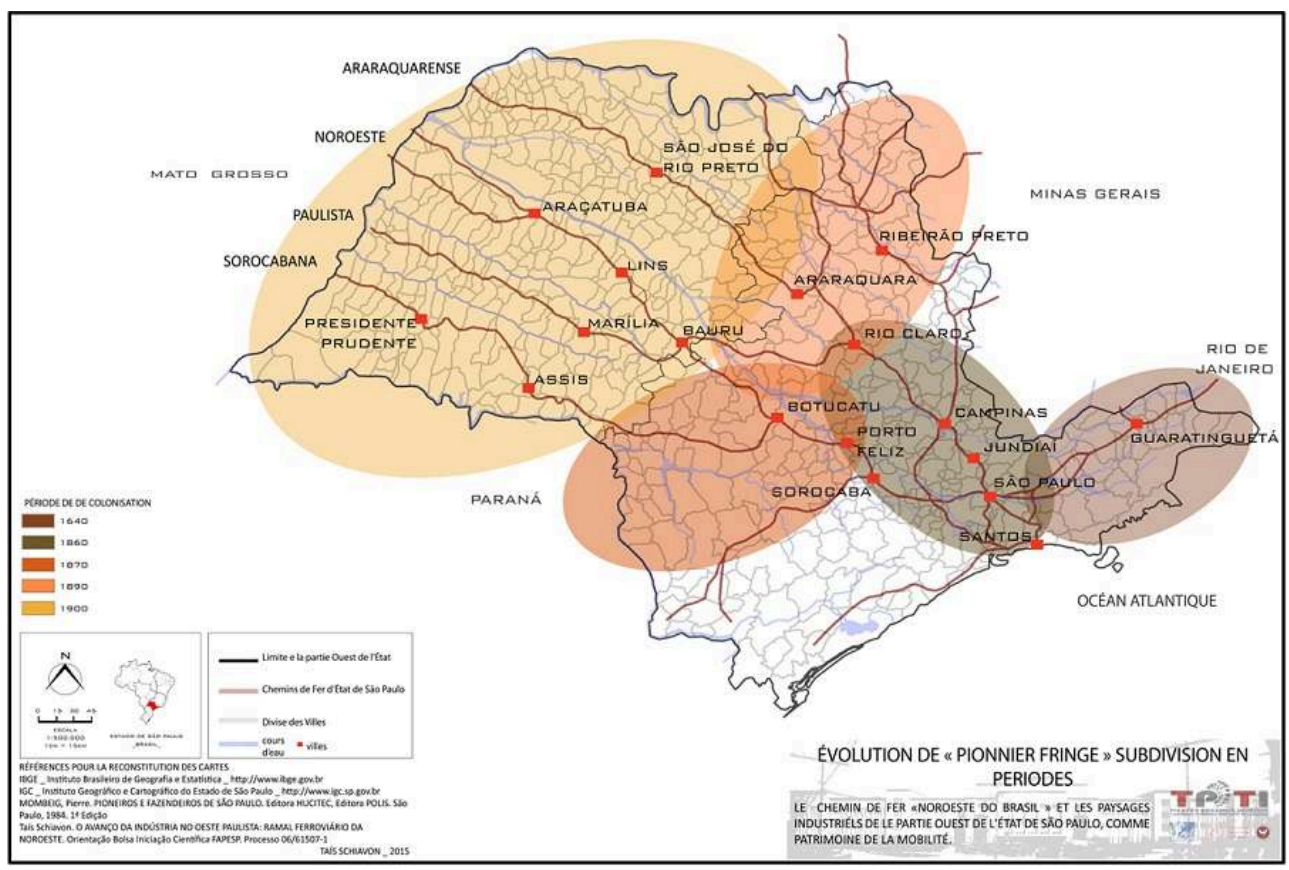

La carte montre le processus d'expansion de l'urbanisation et du développement économique dans l'État de São Paulo. Cette action se produit en chevauchement avec l'avance des principales compagnies ferroviaires. La dynamique de colonisation part de l'État de Rio de Janeiro (Vale do Paraíba,1640), en direction des villes de Jundiaí, São Paulo, Campinas (1860) ; Porto Feliz, Sorocaba et Botucatu (1870) ; Araraquara et Ribeirão Preto (1890) jusqu'à concerner tout l'Ouest de l'État de São Paulo, à partir de 1900.

Source : Schiavon 2005. Réalisé par l'auteur à partir des bases consultées

Au début, le café apporte le développement économique et spatial à l'État, un moyen de colonisation, mais aussi l'impulsion pour la diffusion du réseau d'une grande partie des compagnies de chemins de fer. En 1872, sont créées les Compagnies des chemins de fer Paulista (entre les villes de Campinas et Jundiaí) et Ituana (reliant la ville d'Itu aux lignes de la compagnie São Paulo Railway). Trois ans plus tard, en 1875, sont inaugurées les compagnies Mogiana (reliant les villes de Campinas et Mogi Mirim) et Sorocabana, extension de la compagnie Ituana. En 1896, c'est au tour de la future compagnie Araraquarense (entre les villes d'Araraquara et Ribeirãozinho, actuellement Taquaritinga, et, en 1906, vers la ville de São José do Rio Preto) (Matos 1974).

Le $\mathrm{XX}^{\mathrm{e}}$ siècle peut être caractérisé comme la période d'extension des compagnies de chemins de fer déjà établies, reflétant ainsi la conquête de "l'hinterland inconnu » (Langenbuch 2004), marquée par le territoire qui s'étend à partir des régions urbaines de Lençóis Paulista, Agudos et Piratininga. 


\begin{abstract}
« (...) La « frange pionnière » (...) est une frontière qui progresse de façon irrégulière et dans des directions confuses ${ }^{13}$.» (Monbeig 1984, traduit par l'auteur).
\end{abstract}

$\mathrm{Au} \mathrm{XX}^{\mathrm{e}}$ siècle, les chemins de fer de l'ouest de l'État de São Paulo, représentés par les sociétés Sorocabana, Paulista, Noroeste et Araraquarense, ont assumé la fonction « d'axes de pénétration ", cherchant à développer un territoire et promouvoir sa mise en relation avec les zones centrales du territoire Brésilien. En 1904, la création de la compagnie de chemin de fer Noroeste do Brasil visait à assurer la connexion entre les États du Mato Grosso et de São Paulo. En 1905, les rails ont commencé à être posés dans la ville de Bauru, un emplacement stratégique. La même année, la ville a reçu les rails de la Compagnie Sorocabana et, en 1910, elle accueille également le tracé de la Compagnie Paulista. Le petit village situé en périphérie de l'arrière-pays devient en quelques années une ville d'importance capitale, abritant l'une des plus importantes entrées ferroviaires du pays et de l'Amérique du Sud.

Dans ce processus, la création de nouvelles villes était nécessaire. Les structures urbaines ont été considérées comme un moyen en vue d'aider les compagnies de chemins de fer à assurer le transport des personnes, des marchandises et à garantir des services vers la partie Ouest de l'État, en direction d'autres centres déjà urbanisés. L'ancien paysage des «territoires inconnus » s'est transformé en un espace composé de «villes modernes» créées dans un court laps de temps. La consolidation de la Compagnie Noroeste do Brasil se produit pendant une période d'importance stratégique pour les États concernés par le projet et de manière plus générale pour le Brésil, dans une période par des transitions politiques et une forte instabilité économique.

Contrairement à la plupart des compagnies de chemin de fer du Brésil qui articulent leur expansion sur la culture du café, Noroeste do Brasil a fait de son tracé ferroviaire, axe dédié à la promotion de la colonisation du territoire, pour stimuler la mise en valeur de nouvelles zones économiques et encourager la communication avec l'intérieur du territoire. Pour consolider ce projet, différents moyens sont utilisés, qui visent promouvoir le développement, attirer des travailleurs, les immigrants et les nouveaux investisseurs. Calógeras indique à cet égard que l'analyse du territoire brésilien ne peut pas ignorer la présence d'un régionalisme lié à la logique du système de transport (Calógeras 1928).

Jusqu'au début du $\mathrm{XX}^{\mathrm{e}}$ siècle, le modèle adopté dans le pays pour la diffusion des chemins de fer était proche du système européen, où les compagnies exploitaient des lignes dans des zones déjà peuplées et économiquement développées. Au Brésil, avec l'avancée des rails vers les régions de "l'arrière-pays ", les investissements deviennent plus rares, conséquences de l'incertitude entourant la propagation des compagnies dans les territoires « inconnus et sauvages ».

Le processus d'urbanisation qui a commencé avec l'avancée des routes vers la partie Ouest de l'État de São Paulo est le résultat direct des interférences et influences modernes découlant du déplacement de la « frange pionnière " grâce aux chemins de fer, élément moteur du développement de toute cette région. La compréhension de ce contexte nous permet d'identifier des itinéraires impliqués dans le double mouvement entre l'ancienne colonie et les puissances européennes à travers les compétences nationales et internationales ainsi que les nouvelles potentialités de liens économiques, 
du fait des difficultés techniques rencontrées dans la mise en œuvre d'un projet aussi ambitieux, par son caractère international.

$\mathrm{Au}$ Brésil, le chemin de fer fut la première forme d'ouverture. Il est perçu comme un vecteur de propulsion territoriale et industrielle, qui a soutenu le transfert des marchandises et des travailleurs entre les zones d'expansion et le marché de consommation. Le chemin de fer a fait apparaître des nouveaux éléments structurels et urbains sur tout le territoire. Les bâtiments témoignent encore aujourd'hui de cette période. Vencovsky décrit l'existence de périodes de flexibilité autour des politiques de transports brésiliens. À partir de ces stratégies, l'État s'est d'abord préoccupé de l'occupation du territoire brésilien, puis de sa mise en valeur, avant, enfin, d'inscrire sa démarche dans un cadre international (Vencovsky 2006).

Après cette période, le rythme d'expansion des entreprises brésiliennes a été considérablement réduit du fait de l'instabilité internationale dans un pays encore sous dépendance technique et économique. Certains auteurs voient dans les années 1920 le début de la baisse du transport ferroviaire au profit de l'émergence du modèle routier, dont l'apogée se situe en 1960.

Fig.6. État de São Paulo : expansion des Chemins de Fer

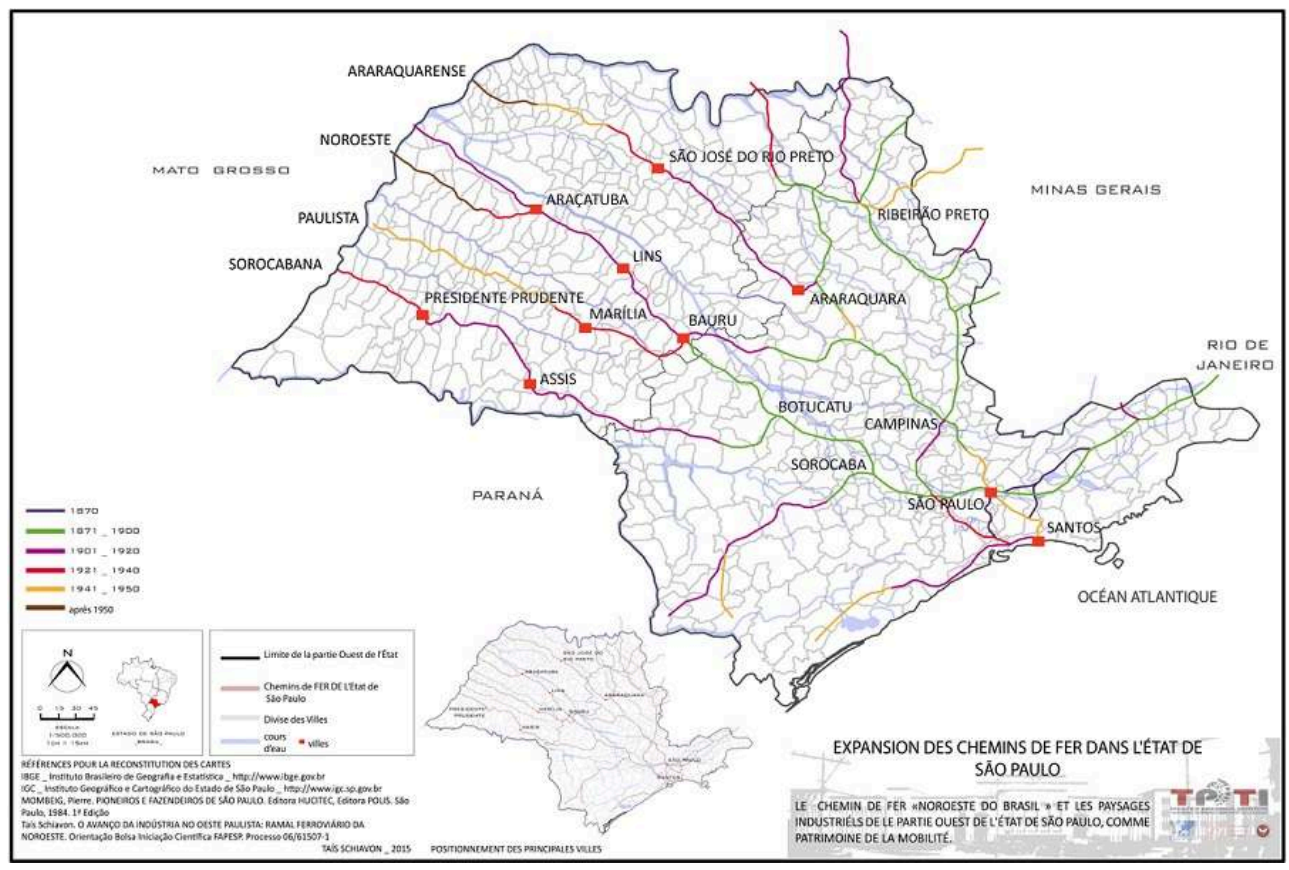

La carte met en évidence le positionnement des principales villes de l'État de São Paulo, articulé par leurs sociétés ferroviaires respectives, dont les périodes d'installation ont des nuances différentes, variant selon des décennies de concentration.

Source : Schiavon 2005. Réalisé par l'auteur à partir des bases consultées

L'analyse des deux principaux moyens de mobilité au Brésil, considérés à partir de leurs différents cycles, démontre que le chemin de fer a été un facteur important pour le développement de l'économie du café et pour la diversification agricole du pays. Le modèle routier est arrivé à une période marquée par l'intense processus $d$ 'industrialisation et des exigences croissantes en termes de communication au sein du territoire national. Le système ferroviaire, au cours d'un intervalle de temps relativement court, est devenu le précurseur d'une « industrie moderne ». 


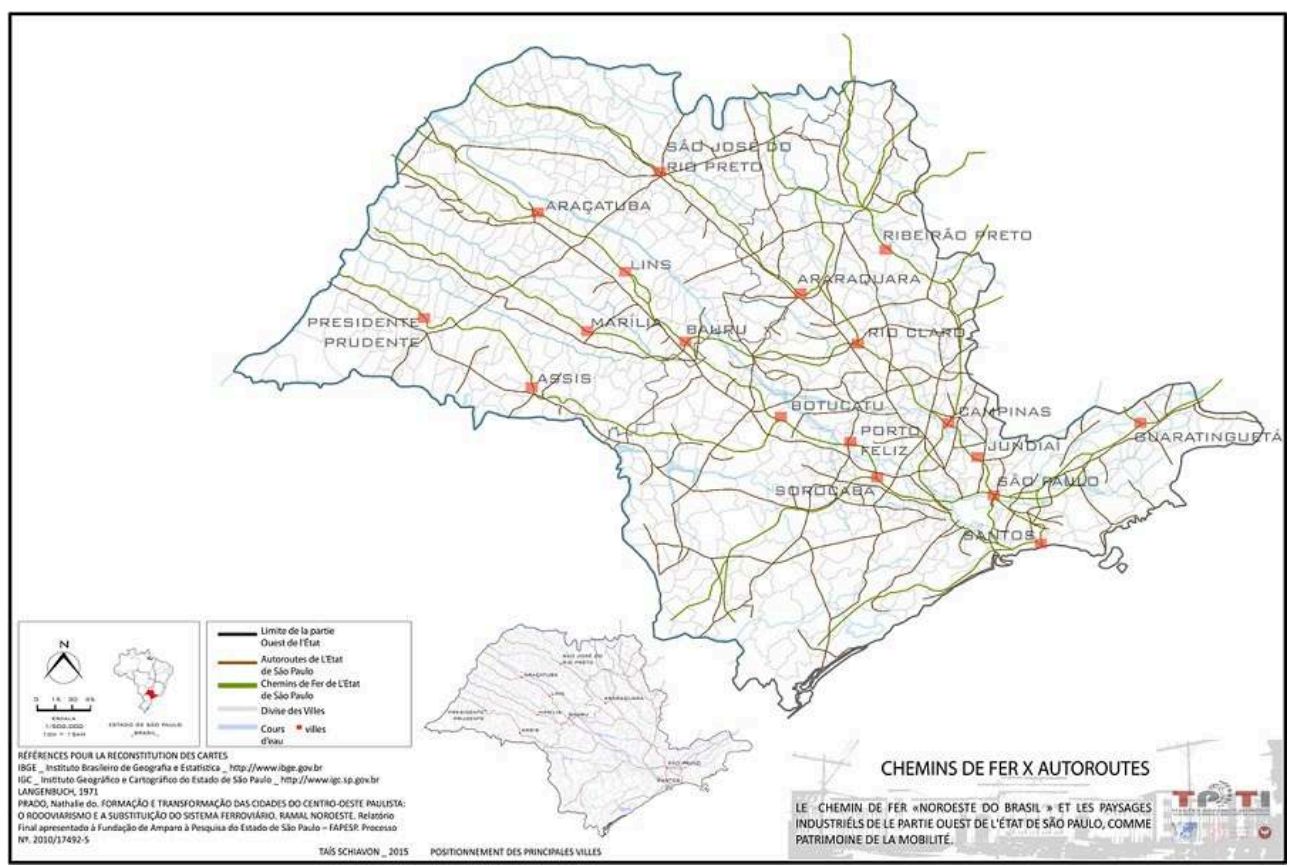

La carte met en évidence le positionnement des principales villes de l'État de São Paulo, illustrant le chevauchement entre les itinéraires des principales compagnies ferroviaires et axes routiers de l'État. Source : Schiavon 2005. Réalisé par l'auteur à partir des bases consultées

Territoire à découvrir, la portion Ouest de l'État de São Paulo voit son expansion s'inscrire dans le cadre des stratégies autour de connexions territoriales nationales et internationales. Dans ce cadre, le système ferroviaire de transport est peu à peu remplacé par le modèle routier. Une nouvelle logique urbaine se dessine alors, qui oblige à repenser les villes précédemment desservies par les rails. Dans cette transition, les anciens symboles de la mobilité et de sa dynamique urbaine entrent en état d'inactivité, ce qui en fait un objet d'étude pour de nouveaux usages (Schiavon 2009).

\section{La production industrielle et le développement économique}

L'expansion industrielle de l'État de São Paulo avant le XIX ${ }^{e}$ siècle est mal connue. Ses origines proviennent des unités agricoles, destinées à la production de sucre, de café et de coton. Dans son analyse des causes de l'industrialisation brésilienne, Cano prend en considération le territoire et les structures commerciales archaïques, pour souligner la difficile tâche de réglementer les chemins de fer au Brésil, mais sans introduire tous les processus industriels nécessaires en vue de répondre aux besoins temporaires des compagnies de chemin de fer comme des Brésiliens. 
Fig.8. Zones de production et dispersion ferroviaire au Brésil au $\mathrm{XX}^{\mathrm{e}}$ siècle

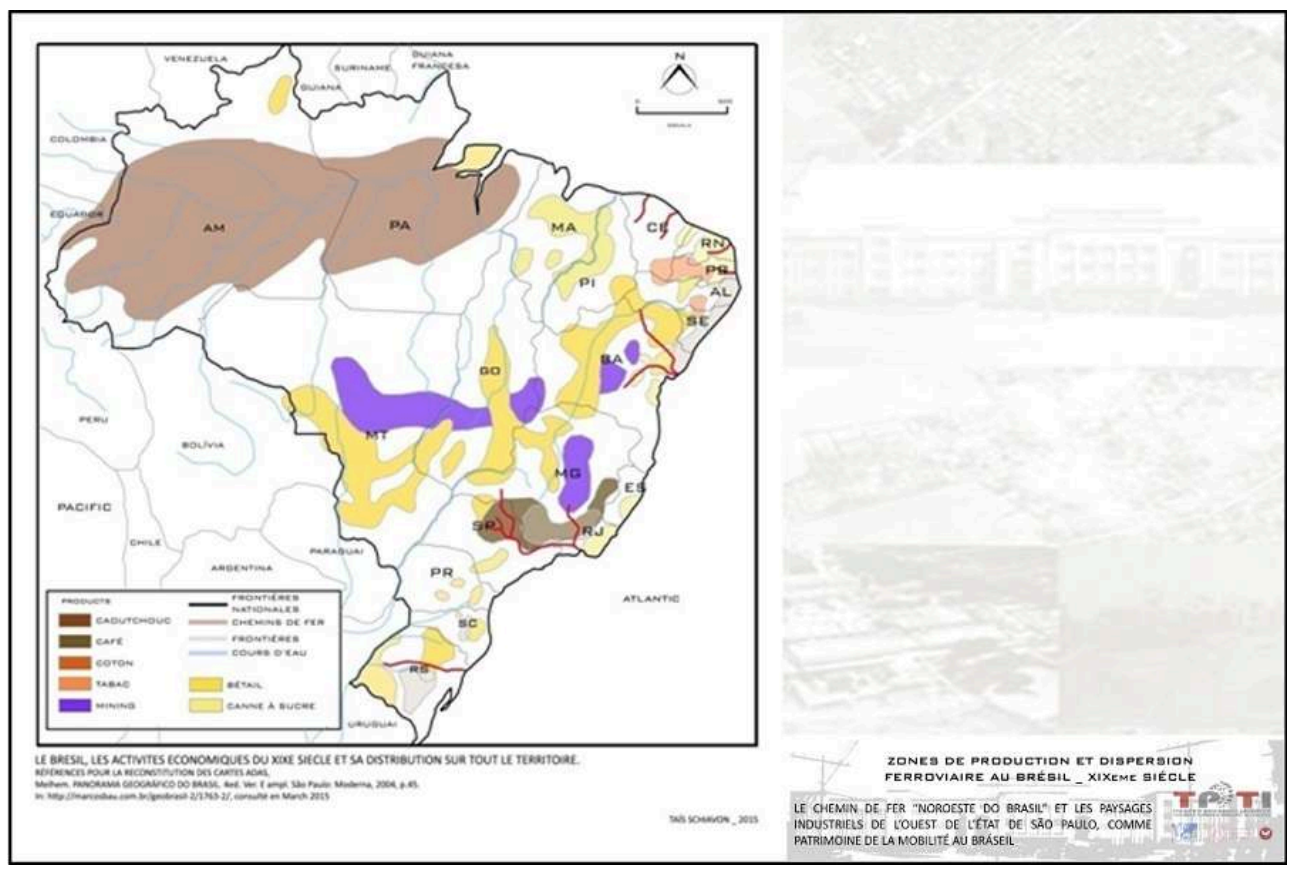

La carte montre la concentration des principales activités agricoles au Brésil jusqu'au XXe siècle et sa correspondance avec la concentration des principaux chemins de fer opérant jusque-là.

Source : Schiavon 2005. Réalisé par l'auteur à partir des bases consultées

En 1907, le premier recensement industriel mené au Brésil confirme l'existence de petites entreprises individuelles, qui produisaient des chapeaux, des chaussures, de la nourriture, des boissons, des meubles, du bois d'œuvre. On note aussi la présence de petits ateliers métallurgiques et d'industries liées à la fabrication de produits chimiques: savon, bougies, médicaments, etc. On trouve aussi, à cette époque, des machines de transformation (café, riz, oléagineux) ainsi que des usines de textile.

$\mathrm{Au}$ début du XIX ${ }^{\mathrm{e}}$ siècle, le complexe du café stimule l'industrie par la transformation des produits récoltés dans les fermes ou dans leurs environs. L'industrialisation est alors représentée par trois segments : la production d'équipements par le traitement du café, l'industrie du jute pour l'emballage des céréales et l'industrie manufacturière. De nouvelles activités s'y ajoutent : scieries, poteries et fonderies apparaissent tout au long de l'avancée de l'axe, notamment dans les villes «bouches de l'arrière-pays » pour répondre à la pénurie de produits à mesure de l'avancée des rails. 
Fig.9. Développement industriel dans l'ouest de l'État de São Paulo

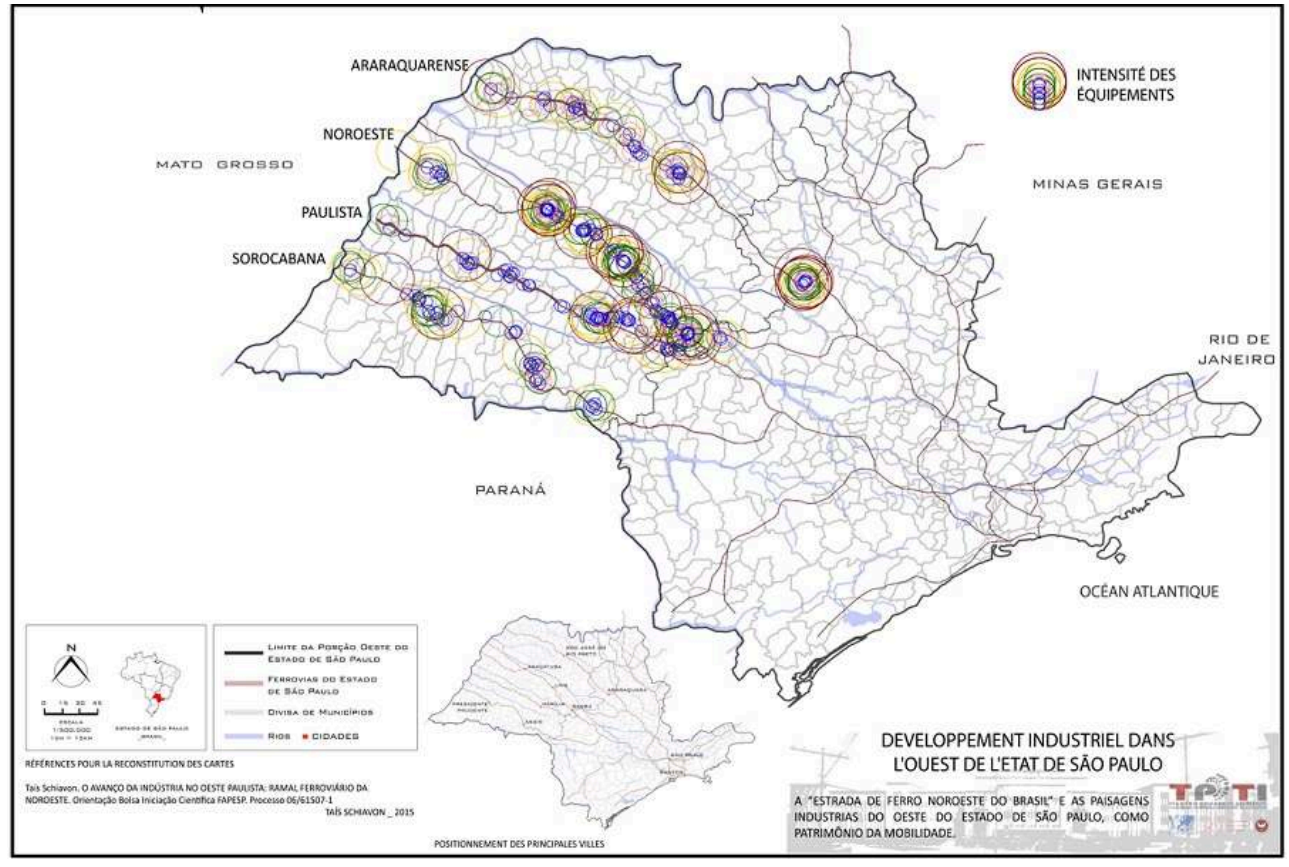

La carte montre la concentration des équipements identifiés tout au long des recherches menées au Brésil, parmi les villes analysées par les compagnies ferroviaires Paulista, Sorocabana, Araraquarense et Noroeste.

Source : Schiavon 2005. Réalisé par l'auteur à partir des bases consultées

Dans les années 1930, le centre de la production de l'État de São Paulo a été déplacé sous l'influence du chemin de Fer de la Noroeste, réaffirmant l'importance de cette région. Ainsi, la ville de Bauru est devenue pour certains intellectuels la nouvelle capitale administrative de l'État de São Paulo, en raison de son positionnement central et de son poids économique, la plaçant parmi les plus importantes villes du maillage ferroviaire de l'État.

\section{Les conséquences urbaines}

Le potentiel économique de la zone de la Noroeste est devenu réalité avec le développement $\mathrm{du}$ chemin de fer qui a fourni les ressources nécessaires à la formation et la croissance des villes et stimulé leur production de biens et services à l'intention des marchés locaux, régionaux, nationaux voire internationaux (Galvao 1996 :172). Aucune autre région d'Amérique du Sud n'a connu une transformation aussi large et profonde, dans un temps aussi rapide : moins de dix ans (Azevedo 1950).

Créées principalement au début du $\mathrm{XX}^{\mathrm{e}}$ siècle, les villes de cette région possèdent une composition urbaine homogène. Durant ce processus, le déploiement des villes de la première moitié du $\mathrm{XX}^{\mathrm{e}}$ siècle peut être caractérisé comme une étape importante de la planification urbaine brésilienne, en particulier en prenant en compte le mouvement dans la région ouest de São Paulo. L'objectif principal tournait autour de l'obtention d'un retour rapide sur les investissements pour les agriculteurs, les entreprises ferroviaires mais aussi pour les compagnies de « colonisation » (Menezes 2008). 


\begin{abstract}
«La structure de la terre a été organisée en lien avec la coexistence de grandes et de petites fermes d'exploitation (...) par le système d'utilisation des terres, par la culture spéculative, qui ont été orientés vers l'exportation du café au début et du coton plus tard. (...) Ceux-ci ont été concernés par la fragmentation d'un plus grand nombre de propriétés et de propriétaires, attirant principalement les immigrants des anciennes zones de production du café ${ }^{14}$ ", (Carvalho 2007, traduction effectuée par l'auteur).
\end{abstract}

37 Cette pratique bénéficie d'incitations, notamment après la promulgation de la loi fédérale des années 1907, avec la dispersion de nombreuses entreprises nationales et internationales, qui liaient les compagnies de chemin de fer, responsable pour la « déchiquetage » de plusieurs fermes situées sur le trajet de la voie ferrée.

"(...) la monotonie du paysage urbain rend répétitif sur le plan géométrique les nouvelles villes (...). Ce sont les circonstances historiques de la fondation qui imposent des changements dans la disposition du tissu urbain (...), nécessitant l'étude des acteurs sociaux de ces sociétés en mouvement ${ }^{15}{ }^{\prime}$ (Monbeig 1998, traduction effectuée par l'auteur).

L'idéal urbain porté par les villes aménagées avec l'avancement de la «frange pionnière " a comme caractéristique l'orthogonalité des quartiers et autres subdivisions. La géométrie du plan d'urbanisme souligne la relation entre les solutions architecturales et ses possibles utilisations, prenant les traits d'un « système moderne » d'organisation urbaine. La dualité qui existe entre la création de nouveaux centres de production et la concentration urbaine montre aussi la nécessité de la permanence de la structure agraire pour aider le développement urbain (Retto 2011). 
Fig.10. Spatialisation urbaine de la Ville de Bauru

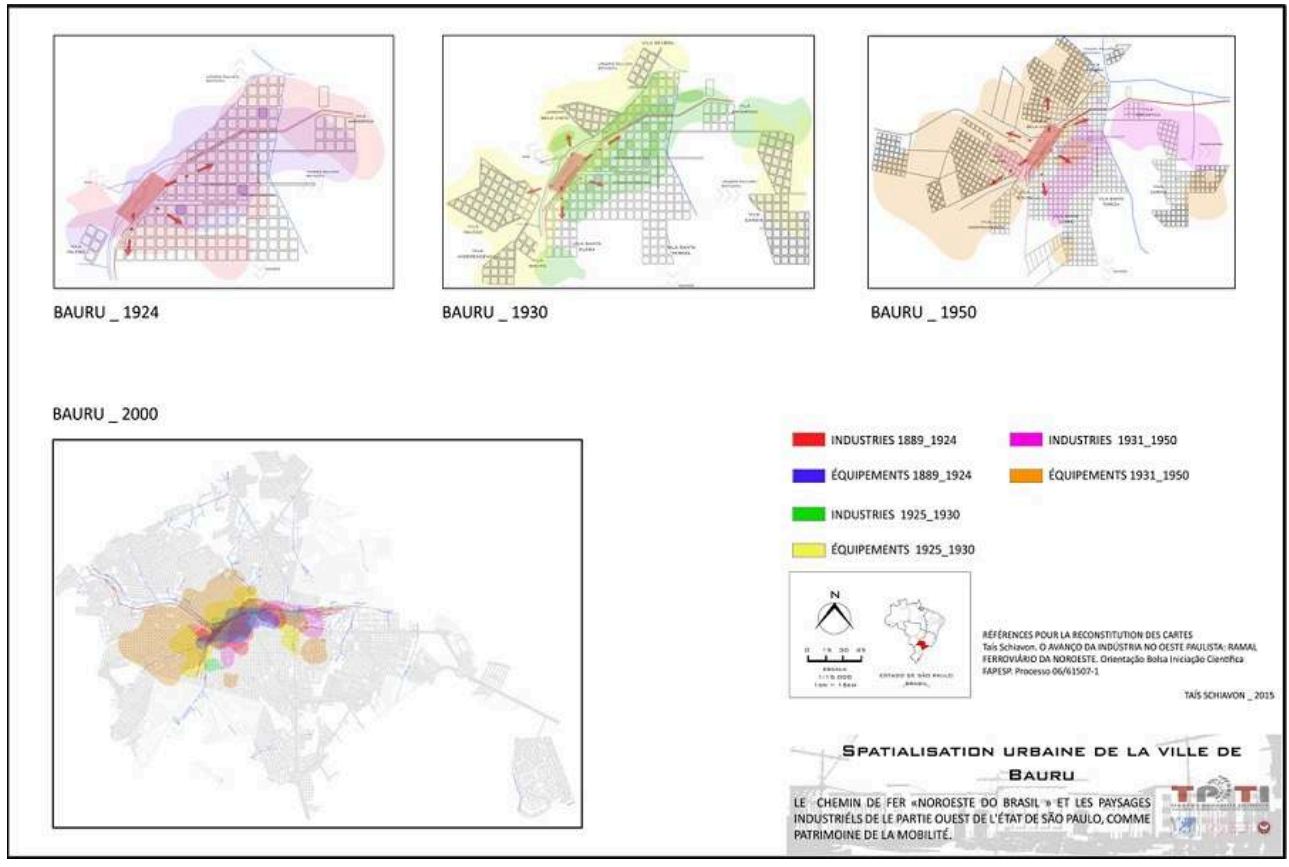

Les différentes cartes montrent la croissance urbaine de la ville de Bauru, entre 1924 et les années 2000 en soulignant la concentration des équipements et des industries à chaque période. II est intéressant de noter qu'une grande partie de la concentration des équipements sur les périodes analysées était concentrée dans les zones proches de l'emprise des compagnies ferroviaires opérant dans la ville.

Source : Schiavon 2005. Réalisé par l'auteur à partir des bases consultées

La gare révèle alors le lien entre l'économie rurale, la compagnie ferroviaire et le contexte urbain. Quand les localités disposent d'axes de développement économique, le tracé urbain perd sa caractéristique initiale marquée par l'homogénéité de son dessin lié à l'importance de la gare. Des avenues commencent à caractériser le nouveau territoire. Cette évolution paraît absente dans les villes moins développées, où la composition urbaine reste le plus souvent inchangée et ceci jusqu'à aujourd'hui. 
Fig.1 1. Les compagnies ferroviaires dans la ville de Bauru

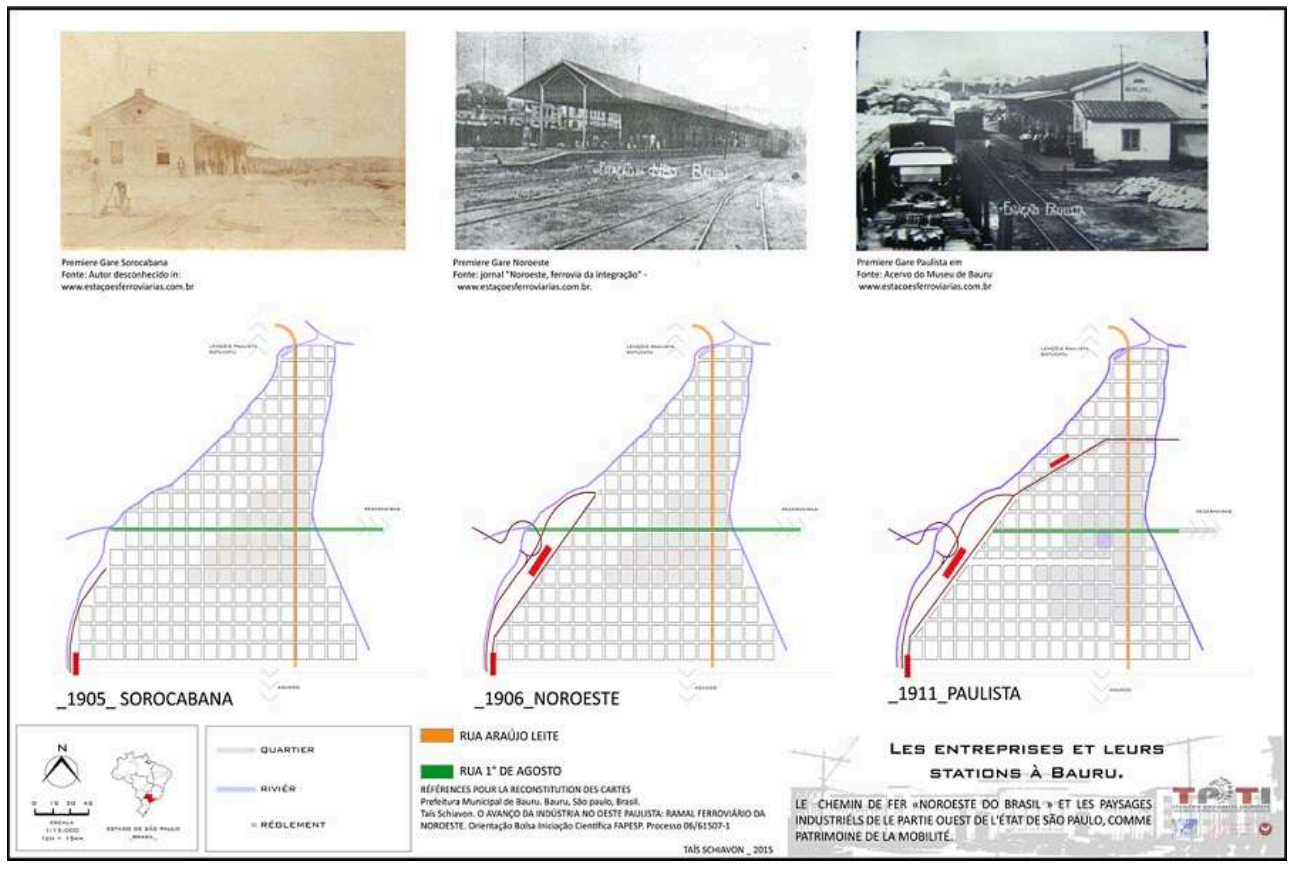

Les cartes montrent l'emplacement et la période d'installation de chaque compagnie ferroviaire opérant dans la ville de Bauru. Les photographies donnent à voir les enregistrements des premières stations de chaque entreprise

Source : Schiavon 2005. Réalisé par l'auteur à partir des bases consultées

40 Avec le temps, les défis entourant l'extension de la zone Noroeste présentent des résultats positifs, favorisant l'intégration de toute la région à l'économie de l'État de São Paulo. Un parcours a permis de constituer une chaîne de villages et de villes toute au long de la ligne du chemin de fer.

\section{Conclusion}

41 Les compagnies ferroviaires fondées au cours du $\mathrm{XX}^{\mathrm{e}}$ siècle dans les régions de « l'arrière-pays », qu'ils s'agissent de Sorocabana, Paulista, Noroeste ou Araraquarense, sont à l'origine de véritables "axes de pénétration ", recherchant le développement d'un territoire "peu peuplé ", grâce à la communication avec les zones centrales du territoire brésilien. Ce mouvement est associé au développement agricole, qui avait comme particularité la diversification de la production, en partenariat avec le développement du café.

Lorsqu'elle s'insère dans un environnement, une compagnie de chemin de fer a besoin d'un réseau de structures actives. Pour pallier l'insuffisance de structures urbaines dans la région ouest de l'État de São Paulo, les compagnies se trouvèrent dans la nécessité de fonder de nouvelles villes ou de renforcer les centres urbains existants. Les géomètres et les ingénieurs organisèrent le parcellaire en ce sens. À partir de la gare, les villes bénéficièrent d'un véritable tracé avec l'établissement de places et d'avenues.

Dans de nombreux cas, la diversification technique et technologique des industries est réalisée à partir de l'avancée des entreprises du chemin de fer. La nécessité de réparer les machines et les équipements ferroviaires a débouché sur la construction des bâtiments, d'installations. On pense aux ateliers, aux petites fonderies dispersées dans 
les centres urbains. Il en découle une diversité technique de la région. En changeant les paysages, les voies de communication représentées par les chemins de fer ajoutent de nouvelles fonctionnalités et intensifient les modifications déjà apportées aux environnements. Les chemins de fer nationaux, en particulier ceux qui se situent dans la partie ouest de l'État de São Paulo, sont les témoins du passé industriel développé à partir du maillage ferroviaire et de l'armature urbaine qui en a découlé. Ces transformations se reproduisent à l'identique à partir des années 1960, autour du transport routier, cette fois.

Fig.12. L'emprise ferroviaire dans la ville de Lins.

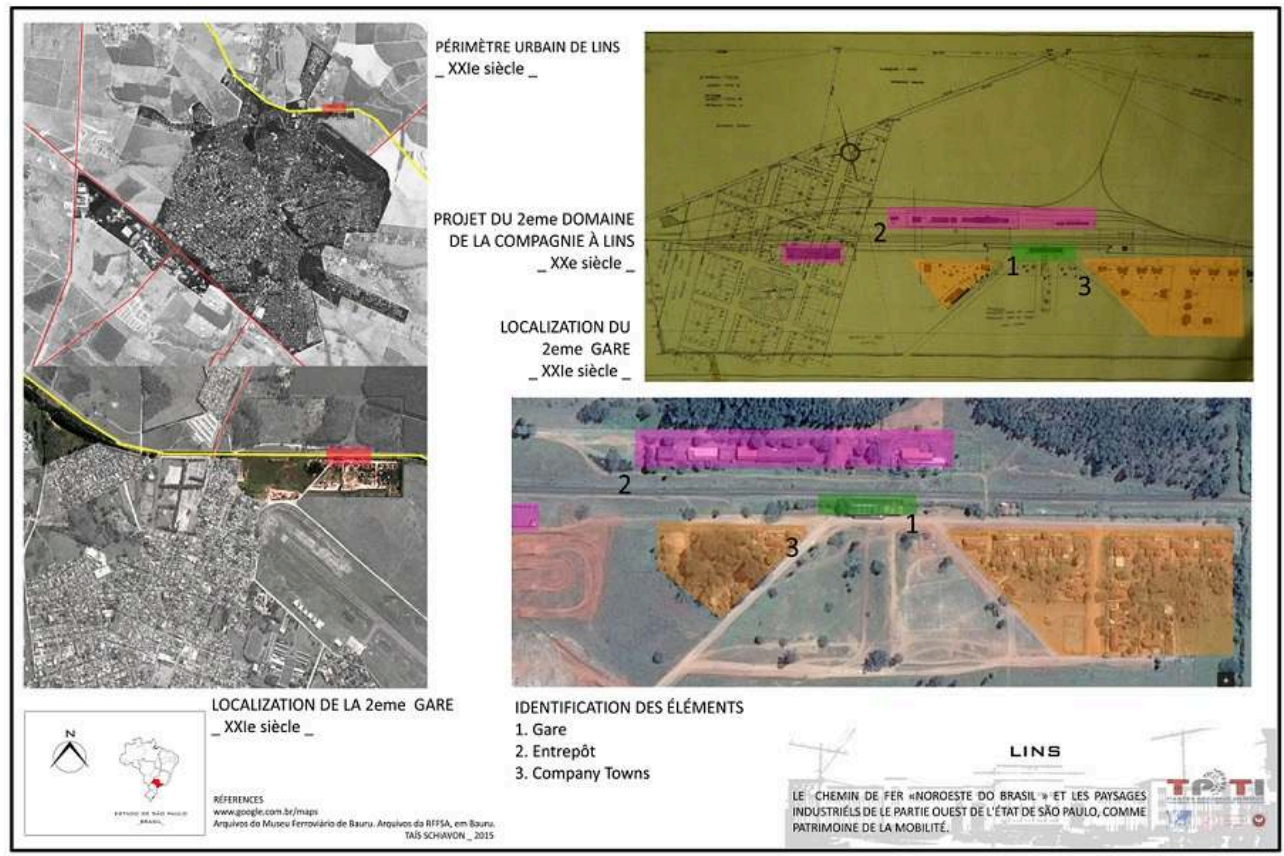

La figure montre le chevauchement des axes ferroviaire (jaune) et autoroutier (rouge), mettant en évidence l'emplacement du chemin de fer Noroeste do Brasil. Les cartes montrent le positionnement de la gare (1), de l'entrepôt de fret (2), et de la cité ouvrière (3).

Source : Schiavon 2005. Réalisé par l'auteur à partir des bases consultées

44 La croissance de l'économie brésilienne n'a pas été accompagnée du processus de modernisation de ses bases dans le cadre des nouvelles exigences de communication territoriales. La nécessité croissante autour de la souplesse et d'efficacité du modèle ferroviaire se manifeste dans les périodes marquées par une forte intervention de l'État, qui finit par considérer le modèle routier comme le nouvel idéal de développement du pays. Il met ainsi en péril la préservation des chemins de fer et favorise le processus de dégradation du paysage urbain.

La transition des types de communications territoriales s'articule sur de nouveaux points, fondés sur différentes notions de vitesse et d'espace. Avec cette nouvelle dynamique, le milieu urbain commence à prendre une nouvelle organisation, se superposant aux modèles précédents (Ascher 2010). À cet égard, le patrimoine industriel créé autour de ce processus de transition comprend les traces d'une culture industrielle, établie comme résultat du chemin de fer. Il est composé d'environnements chargés de nombreuses valeurs historiques, technologiques, sociales, architecturales et scientifiques, à travers les bâtiments, les machines, les ateliers, les entrepôts. Peuvent 
aussi être prises en considération les installations de production, de transmission et l'utilisation de l'énergie, couplées aux moyens de transport, ainsi que les lieux où se sont développées les activités sociales liées à l'industrie (Kuhl 2008).

Fig.13. L'emprise ferroviaire dans la ville d'Araçatuba

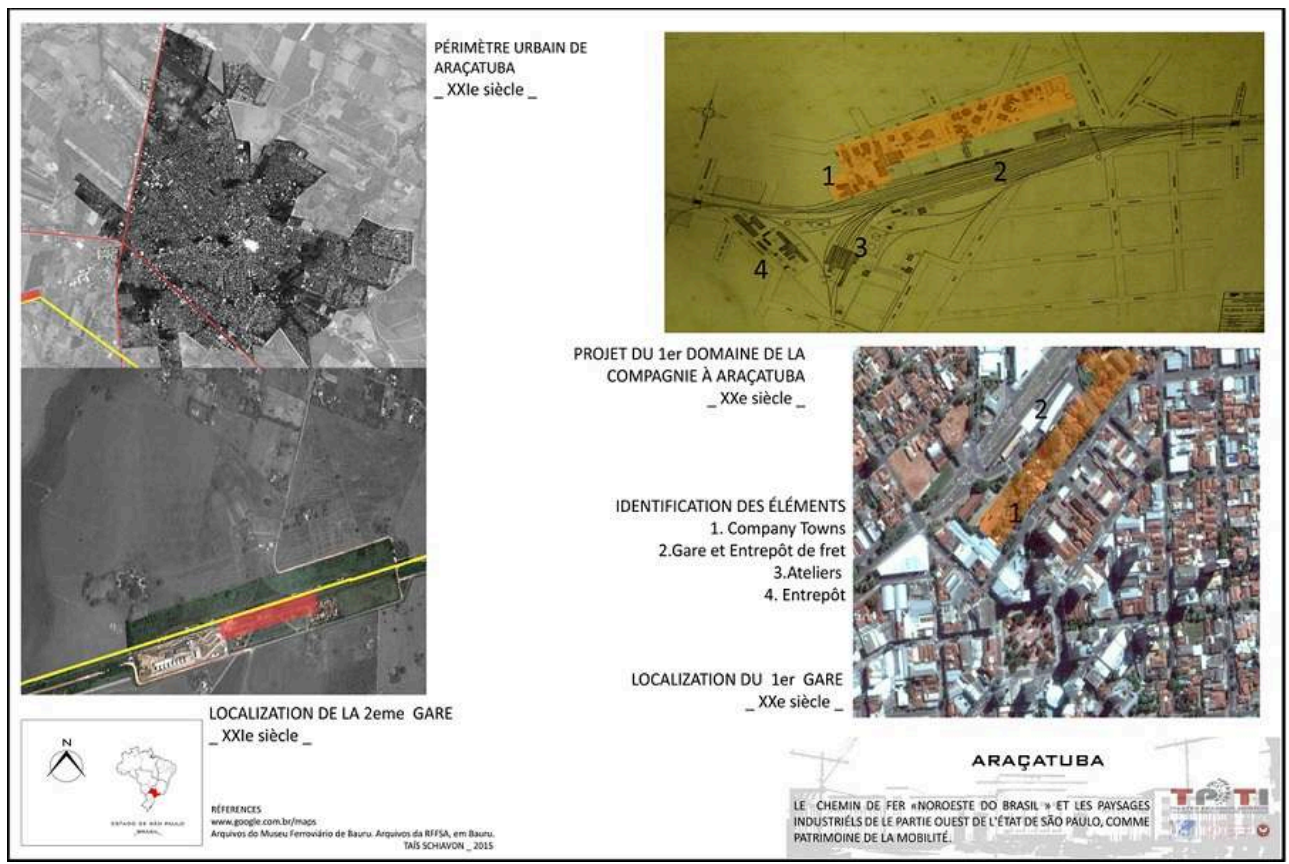

La figure montre le chevauchement des axes ferroviaire (jaune) et autoroutier (rouge), mettant en évidence l'emplacement du chemin de fer Noroeste do Brasil. Les cartes montrent le positionnement de la cité ouvrière (1), de la gare et de l'entrepôt de fret (2), des ateliers (3) et de l'entrepôt

Source : Schiavon 2005. Réalisé par l'auteur à partir des bases consultées

Contrairement aux pratiques internationales caractérisées par l'entretien et la rénovation des installations ferroviaires et par la réutilisation des gares pour résoudre les problèmes locaux et régionaux, ce processus a été largement négligé au Brésil à quelques exceptions près. Les anciennes installations se sont dégradées. Or, ces installations représentent au sein du contexte urbain des éléments d'importance historique, susceptibles d'être réhabilité. Les compagnies ferroviaires «ont joué des rôles différents selon les époques, (...). Ces équipements sont alors des vitrines du savoir-faire ferroviaire (...) et aussi ont contribué au développement de lotissements suburbains (...). L'essor du ferroviaire a donc beaucoup marqué l'histoire du territoire et les gares sont aujourd'hui porteuses de cet héritage » (Bowie 2015).

En ce qui concerne la législation, le modèle brésilien présente, comme en France, une profonde réflexion sur certains aspects de préservation, impliquant une approche historique, sociologique et anthropologique. Mais la législation et les procédures de conservation et de réhabilitation sont beaucoup plus codifiées en France qu'au Brésil (Kuhl 2008). L'élaboration d'une véritable programmation devrait articuler les projets architecturaux et d'aménagement paysager autour des caractéristiques morphologiques de la région étudiée, en les combinant avec leur histoire et les pratiques locales, à partir du principe suivant : « (...) La préservation et la rénovation des bâtiments, des districts et des paysages historiques affirment la continuité et l'évolution de la société urbaine ${ }^{16}{ }^{\prime}$. 
imoine de la mobilité par excellence, une industrie ferroviaire implique non seulement des stations, mais aussi tout un environnement économique, qui met en œuvre des aménagements urbains, des anciens centres d'importance autour de la production agricole et industrielle, de la distribution de ses produits, le tout reposant sur des compétences techniques développées dans un cadre national mais aussi international.

\section{BIBLIOGRAPHIE}

ACIOLI Rodrigo Girdwood, Os Mecanismos de financiamento das Ferrovias Brasileiras, Dissertação Universidade Federal do Rio de Janeiro, 2007

ALMEIDA F.F.M, Fundamentos geológicos do relevo paulista, São Paulo, IGEOG, 1974

ASCHER F., Os Novos Preceitos do Urbanismo, São Paulo, Romano Guerra, 2010

AZEVEDO Fernando de, Um Trem corre para o Oeste. Estudo sobre a Noroeste e seu papel no sistema de Viação Nacional, São Paulo, Livraria Martins Editora, 1950

BOWIE Karen, Les grandes gares parisiennes au XIX ${ }^{e}$ siècle. Paris, Action artistique de la Ville de Paris, 1987

BRESCIANI Maria Stella Martins, RETTO JUNIOR Aldaberto da Silava, « Utopia, cidade, território : da França ao Brasil » Colóquio Internacional Interdisciplinar Pontes \& Idéias Luois-Leger Vauthier engenheiro francês no Brasil. Recife, Caderno de programação e resumos, Recife, 2009

BRESCIANI Maria Stella Martins, Saberes eruditos e técnicos na configuração e reconfiguração do espaço urbano : Estado de São Paulo, séculos XIX e XX, São Paulo, 2006

CALÓGERAS João Pandiá, Pandiá, Formação Histórica do Brasil, Brasilania, 1938

CALÓGERAS João Pandiá, Problemas de Governo, São Paulo, Empresa Graphica Rossetti LTDA, 1928

CARVALHO Marina Barroso de, Conformação da Estrutura Agrária na formação do tecido urbano das cidades do Oeste Paulista : Linha Noroeste, Orientação Bolsa Iniciação Científica, Processo 06/58402-3, 2007

GALVÃO Dora da Silva Ferreira, A expansão geopolítica ferroviária para a região Noroeste do Brasil até a década de 40, São Paulo, 1996

KARVAR Anouar, «Les élèves étrangers. Analyse d'une politique », in BELHOSTE Bruno, MASSON Francine, PICON Antoine (dir.), Le Paris des Polytechniciens : des ingénieurs dans la ville 1794-1994, Paris, Collection Paris et son Patrimoine, Délégation à l'Action Artistique de la Ville de Paris, 1994, p. 417-434

KUHL Beatriz Mugayar, Preservação do Patrimônio Arquitetônico da Industrialização, Problemas Técnicos de Restauro, Ateliê Editorial, Cotia, São Paulo, 2008

LANGENBUCH Juergen Richard, « Ferrovias e cidades do Oeste Paulista », in RETTO Jr., AS. ENOKIBARA M., CONSTANTINO N. R. T., MAGALHÃES, K. C. (dir.), Conhecimento Histórico-Ambiental Integrado na planificação territorial e urbana. São Paulo, Cultura Acadêmica, 2011 
MATOS Odilon Nogueira de, Café e Ferrovias. A Evoulção Ferroviária de São Paulo e o Desenvolvimento da Cultura Cafeeira, São Paulo, Alfa-Omega, 1974

MONBEIG Pierre, Pioneiros e Fazendeiros de São Paulo, São Paulo, Editora HUCITEC, Editora POLIS, (1984) 1998

PINTO Adolfo, Istória da viação pública de São Paulo, São Paulo, Tip. de Vanordem, 1903

PRADO Nathalie do, Formação E Transformação Das Cidades Do Centro-Oeste Paulista : O Rodoviarismo e a substituição do Sistema Ferroviário. Ramal Noroeste do Brasil, BP.IC. Processo, 2011

RETTO JR Adalberto da Silva, « A criação de um acervo virtual nas cidades Bocas do Sertão como patrimonio da mobilidade. Por um "Conhecimento Histórico Ambiental Integrado na Planificação Territorial e Urbana.", In V Congresso Internacional de História e Patrimônio Ferroviário "Os legados para nosso futuro", 2020

RETTO JR Adalberto da Silva, ENOKIBARA Marta, CONSTANTINO Norma R. T., « The grid and its variations on the extensive occupation of the West of São Paulo State: A comparative study on the four Railroads ", in 15th International Planning History Society Conference. Cities, Nations and Regions in Planning history, 2011

SALGUEIRO Heliana Angotti (org), Pierre Monbeig e a Geografia Humana Brasileira, Bauru, EDUSC, 2006

SCHIAVON Taís, Documentação iconográfica e bibliográfica dos profissionais e personagens presentes nas cidades formadas com a abertura das Zonas Pioneiras no Oeste do Estado de São Paulo, PC.TT. Processo, 2011

SCHIAVON Taís, Le Chemin de Fer Noroeste do Brasil et les paysages industriels de l'Ouest de l'État de São Paulo, comme patrimoine de la Mobilité au Brésil, Master TPTI, Universidade de Évora, Évora, Portugal, 2015 ; URL : http ://dspace.uevora.pt/rdpc/handle/10174/18401

SCHIAVON Taís, O avanço da indústria no Oeste Paulista : Ramal Ferroviário da Noroeste, BP.IC. Processo, 2009

SCHIAVON Taís, A Noroeste do Brasil e as paisagens Industriais do Oeste de São Paulo. A conformação do Patrimônio da Mobilidade no Brasil, Novas Edições Acadêmicas, 2018

TICCIH, Charte de Nizhny Tagil pour le patrimoine industriel, 2003 ; URL : http:// www.mnactec.cat/ticcih/pdf/NTagilPortuguese.pdf, consulté en septembre 2014.

\section{NOTES}

1. «Saberes eruditos e técnicos na configuração e reconfiguração do espaço urbano : Estado de São Paulo, séculos XIX e XX. AP. TEM. Processo : 05/55338-0 », coordenation prof. " Dr. " Maria Stella Martins Bresciani (UNICAMP). Subtema III - «Saberes Urbanos na configuração e re-configuração das cidades formadas com a abertura de zonas pioneiras no Oeste do Estado de São Paulo », coordenation prof. Dr. Adalberto da Silva Retto Junior (UNESP, Bauru). Le projet impliquait des partenariats entre des universités brésiliennes (UNICAMP, PUC-CAMP e UNESP) et l'IAUV de Venezia. La coordination générale était effectuée par la Profa. Dra. Maria Stella Martins Bresciani. Les groupes d'analyse comptaient sur la participation des Profa. Dra. Ivone Salgado, Prof. Dr. Edgar De Decca, Profa. Dra. Cristina Meneguello, Profa. Dra. Silvana Rubino, Prof. Dr. Adalberto da Silva Retto Jr, Profa. Dr. Norma Constantino, Profa. Dr. Marta Enokibara et Prof. Dr. Celio Losnak. À Bauru, la recherche s'est faite grâce à la participation des étudiants: Dieila Niliane Nazario Ribeiro, Fernanda Bragheto Junqueira, Mônica Harumi Yosioka, Mônica Nakatani Pedro, Éverton Pelegrini 
de Menezes, André Luiz Ribeiro,Fernanda de Lima Lourencetti, Malena Rodrigues Alves, Taís Schiavon, Wilson Lopes Christensen Barcellone, Nathalie do Prado, Dâmaris Oliveira Barbosa Rodrigues, Marília Campos Hildebrand, Ana Beatriz Geovani, Lara Alcadipani de Oliveira, Tatiana Aoki Cavalcanti Silva, Thybor Malusá, Verônica Maria Alves Lima, Aline Silva Santos, Ana Beatriz Gasparotto, Giovanna Carraro Maia, Rafael Tadeu Simabuko, Bruna Panigassi Zechinato, Giovanna Carraro Maia Machado, João Felipe Almeida Lança, Juliana Diehl, Rafael Tadeu Simabuko, Eliza Fernandes de Oliveira Bertholdo de Souza, Gabriela Russo Nóbrega, Ludmilla Righi Orsi, Tatiana Machado Traina, Carolina Zequim, Eliane Katayama Pricoli Amaro, Marina Barroso de Carvalho, Rafaela Maria Serafim, Bruna Stéphanie Tácito, Marina Barroso de Carvalho.

2. Les premiers tests en portugais sont en cours sur les plateformes suivantes: https:// patrimoniomobilidade.wixsite.com/patrimoniomobilidade; https://www.facebook.com/ patrimonio.damobilidade.5; https://www.instagram.com/patrimonio_da_mobilidade/; où le projet global fait partie de la recherche : "Conhecimento histórico ambiental integrado na planificação territorial e urbana : a criação de um acervo virtual nas cidades Bocas do Sertão".

3. Nous devons considérer aussi qu'en plus de la compagnie de Chemin de Fer Noroeste do Brasil, cela influence également le développement dans la région Ouest des compagnies : Estrada de Ferro Araraquarense, Companhia Paulista de Estrada de Ferro et Estrada de Ferro Sorocabana.

4. Terme utilisé par Monbeig dans son étude du Brésil, pour appeler les villes ayant la plus grande concentration de ressources et d'indices de développement, en raison du temps d'arrêt supérieur pour l'ouverture de l'entreprise.

5. Terme utilisé par Monbeig et Langennbuch, pour appeler la ville du front des axes d'une compagnie de chemin de fer comme une référence spécifique à la ville dans laquelle l'avancement des pistes est en développement.

6. «Hoje é o caminho de ferro que funda as cidades e promove, com a industria, as grandes aglomeraçôes urbanas (...), hoje cidades de primeira ordem, (...)».

7. Ce projet fait usage de l'analyse des différentes villes coupées par le chemin de fer Noroeste do Brasil, telles que Bauru, Avai, Pirajuí, Penápolis, Presidente Alves, Cafelandia, Lins et Araçatuba.

8. L'expression Ouest de l'État de São Paulo largement utilisée ici correspond à la régionalisation géomorphologique adoptée par Almeida 1974 pour caractériser la région du " plateau Occidental de São Paulo », c'est-à-dire, " la part de l'État de São Paulo située à l'ouest des villes de São José do Rio Preto, Bauru et Ourinhos » (Langenbuch 2004).

9. Ces données peuvent être analysées sur les sites Web: https:// patrimoniomobilidade.wixsite.com/patrimoniomobilidade et https://www.facebook.com/ patrimonio.damobilidade.5.

10. IPHAN (Institut du Patrimoine Historique et Artistique du Patrimoine National), responsable de la conservation des différents éléments qui forment la société brésilienne, de la promotion, de la coordination et de la préservation du patrimoine culturel du Brésil, visant à renforcer son identité et sa mémoire, en collaboration avec le développement socio-économique du pays. L'organisation a été créée le 13 janvier 1937 par le gouvernement de Getúlio Vargas, par la loi $\mathrm{n}^{\circ} 378$.

11. Loi 11.483, 2007.

12. « A expressão 'Oeste Paulista' amplamente utilizada aqui corresponde a regionalização geomorfológica adotada por Almeida (1974) ao caracterizar a região do Planalto Ocidental Paulista, ou como completado por Langenbuch (2004), como "a parte do estado de São Paulo situada para ooeste de São José do Rio Preto, Bauru e Ourinhos". A expressão "áreas pioneiras" corresponde ao termo adotado por Monbeig (1984), em seu estudo clássico sobre Oeste Paulista, para caracterizar as áreas que primeiro foram abertas pelas ferrovias. Vale lembrar, como lembrou Salgueiro (2006), que muito antes das sucessivas levas históricas de pioneiros do século $\mathrm{XX}$, boa parte do território nacional era ainda desconhecida, conforme mostram os mapas nos anos 1910/1912, onde ocorre a inscrição : 'território inexplorado, habitado por índios ». 
13. « (...) 'franja pioneira' (...) é uma fronteira que progride irregularmente e em direções confusas. »

14. «O uso do território pode ser verificado através da implantação de infra-estruturas, da dinâmica da economia e da sociedade, das políticas dos governos e das empresas, das normas e leis utilizadas na regulação, das regras de financiamento e da agricultura ».

15. « (...) monotonia da paisagem urbana, que faz com que se repita em todos a mesma planta geométrica, própria, às cidades novas (...) são as circunstâncias históricas de fundação que impõem modificações na disposição da trama urbana (...) sendo necessário o estudo dos autores sociais desta sociedade em movimento ».

16. The Congress of the New Urbanism; URL : http ://www.cnu.org.

\section{RÉSUMÉS}

La construction du chemin de fer Noroeste do Brasil, située dans l'Ouest de l'État de São Paulo, au Brésil, peut être caractérisée comme un exemple de l'internationalisation des entreprises européennes et brésiliennes dans un territoire encore peu urbanisé. Un mouvement qui coïncide avec la deuxième moitié du XIX siècle, et qui peut être considéré comme le résultat de la réduction de la production du café dans l'État de Rio de Janeiro, autour de nouvelles zones de colonisation. Si l'on considère le fait que $65 \%$ du territoire de l'État de São Paulo a été urbanisé, à partir de l'avancement de la «frange pionnière", nous comprenons l'importance de la performance des compagnies ferroviaires au Brésil, où les compagnies ont promu le transport des personnes, marchandises et services, changeant ainsi l'ancien paysage de «territoires inconnus », pour un environnement composé de villes « modernes », créées dans un court laps de temps. Les centres urbains développés par le chemin de fer démontrent les impacts de la transition vers le modèle routier, qui s'est intensifiée à partir des années 1960. Cela constitue un scénario dynamique, capable de décrire le patrimoine de la mobilité comme un musée à ciel ouvert.

The construction of the Noroeste do Brasil railway, located in the western part of the state of São Paulo, Brazil, can be characterised as an example of the internationalisation of European and Brazilian companies in a territory that was not yet very urbanised. A movement that coincides with the second half of the 19th century, and that can be considered as the result of the reduction of coffee production in the state of Rio de Janeiro, around new areas of colonisation. If we consider the fact that $65 \%$ of the territory of the State of São Paulo was urbanised, starting from the advancement of the "pioneer fringe", we understand the importance of the performance of the railway companies in Brazil, where the companies promoted the transport of people, goods and services, changing the old landscape of "unknown territories", for an environment composed of "modern" cities, created in a short period of time. The urban centers developed by rail demonstrate the impacts of the transition to the road model, which intensified from the 1960s onwards. This constitutes a dynamic scenario, capable of describing the mobility heritage as an open-air museum. 
INDEX

Mots-clés : histoire des techniques, mobilité, chemin de fer, patrimoine industriel, patrimoine ferroviaire, paysage industriel, urbanisation

Thèmes : Horizons internationaux

Keywords : history of technology, industrial heritage, mobility, railway, urbanisation

\section{AUTEUR}

\section{TAÍS SCHIAVON}

Architecte et urbaniste, titulaire du master Erasmus Mundus TPTI, je me suis consacrée ces dernières années à des recherches portant sur des thèmes liés à l'urbanisme et à la compréhension des impacts historiques et actuels liées au processus d'établissement de modèles de transport ferroviaire et routier dans différentes villes et contextes culturels. Dans cette trajectoire d'études, je m'intéresse à la compréhension des impacts techniques et technologiques au milieu des idéaux de la modernité insérés dans chaque projet de transport, cherchant la valorisation des vestiges historiques et la réinsertion des paysages industriels dans les nouveaux contextes urbains. 